\title{
Improved synchronization criteria for fractional-order complex-valued neural networks via partial control
}

\author{
Hong-Li Li ${ }^{1 *} \mathbb{D}$, Ahmadjan Muhammadhaji ${ }^{1}$, Long Zhang ${ }^{1}$, Haijun Jiang ${ }^{1}$ and Zhidong Teng ${ }^{1}$
}

\section{"Correspondence:}

lihongli3800087@163.com

'College of Mathematics and

System Sciences, Xinjiang

University, Urumqi, China

\section{Springer}

\begin{abstract}
In this article, without dividing a complex-valued neural network into two real-valued subsystems, the global synchronization of fractional-order complex-valued neural networks (FOCVNNs) is investigated by the Lyapunov direct method rather than the real decomposition method. It is worth mentioning that the partial adaptive control and partial linear feedback control schemes are introduced, by constructing suitable Lyapunov functions, some improved synchronization criteria are derived with the help of fractional differential inequalities and L'Hospital rule as well as some complex analysis techniques. Finally, simulation results are given to demonstrate the validity and feasibility of our theoretical analysis.
\end{abstract}

Keywords: Synchronization; Fractional-order; Complex-valued neural networks; Partial adaptive control

\section{Introduction}

During the past few years, real-valued neural networks (RVNNs) have attracted much attention due to the background of a wide range of applications such as associative memory, pattern recognition, image processing and model identification [1-6]. Since Pecora and Carroll introduced a method to realize synchronization of two identical chaotic systems with different initial conditions [7], synchronization has become a widely studied topic. Up to now, there are many sorts of synchronization, such as complete synchronization [8], projective synchronization [9], and quasi-synchronization [10]. Correspondingly, several different approaches have been used for achieving synchronization, for example, linear feedback control [11], partial control [12] and adaptive control [13]. It is well known that the advantage of adaptive control is that the control parameters can adjust themselves according to the updating laws, which are designed according to the characteristics of the considered system. As we know, many applications of neural networks refer to complexvalued signals, which cannot be handled well by RVNNs, complex-valued neural networks (CVNNs) are such neural networks that their states, connection weights and activity functions are all complex-valued [14, 15]. Compared with RVNNs, CVNNs have more complicated properties, and can handle complex signals better. In recent years, the study on

(c) The Author(s) 2020. This article is licensed under a Creative Commons Attribution 4.0 International License, which permits use sharing, adaptation, distribution and reproduction in any medium or format, as long as you give appropriate credit to the original author(s) and the source, provide a link to the Creative Commons licence, and indicate if changes were made. The images or other third party material in this article are included in the article's Creative Commons licence, unless indicated otherwise in a credit line to the material. If material is not included in the article's Creative Commons licence and your intended use is not permitted by statutory regulation or exceeds the permitted use, you will need to obtain permission directly from the copyright holder. To view a copy of this licence, visit http://creativecommons.org/licenses/by/4.0/. 
CVNNs has attracted some attention, and some important and interesting results have been obtained [16-18]. In [18], Song et al. studied the global exponential stability for the addressed CVNNs based on Lyapunov direct method.

Compared with integer-order derivative, fractional-order derivatives can better depict processes and materials possessing hereditary and memory characteristics [19-21], these characteristics make fractional-order systems promising candidates for describing some real-world phenomena [22, 23]. Some recent studies regarding synchronization control and optimal control for fractional-order systems may be found in [24-26]. Lately, many researchers consider fractional-order complex-valued neural networks (FOCVNNs), and some remarkable results on bifurcation [27] and stability [28] as well as synchronization [29-31] have been reported. We note that the key technique in [27-31] is to transform the considered FOCVNNs into two equivalent real-valued subsystems, and then their dynamics are studied by employing methods of dealing with fractional-order real-valued neural networks (FORVNNs), that is, the real decomposition method. In [32], Li et al. designed a linear feedback controller and adaptive controller, and the complete synchronization and quasi-projective synchronization criteria for FOCVNNs were derived by using the Lyapunov direct method, respectively.

To the best of our knowledge, a neural network may have a large amount of neurons, it is impossible and unnecessary to impose controllers on all neurons in such large-scale network. To reduce the number of controlled neurons, partial control should be considered. Till now, partial control for neural networks remains little investigated [33, 34]. In [34], Wu et al. discussed the stability control for FORVNNs by employing partial linear feedback control strategy. However, to the best of the authors' knowledge, there is no result concerning synchronization problem for FOCVNNs by using partial control scheme. Motivated by the above discussions, this paper investigates the global synchronization for the addressed FOCVNNs by partial control. The main contributions of this paper are the following three aspects: (1) The partial adaptive control and partial linear feedback control schemes are first proposed to realize synchronization of FOCVNNs. (2) FOCVNNs are investigated by Lyapunov direct method rather than real decomposition method, which can reduce computational complexity. (3) Several succinct synchronization criteria for FOCVNNs are derived.

The organization of the paper is as follows. In Sect. 2, the model formulation and preliminary results are presented. In Sect. 3, the partial adaptive control and partial linear feedback control schemes are proposed to achieve synchronization for the addressed FOCVNNs. In Sect. 4, numerical simulations are provided to illustrate the effectiveness of our theoretical results. Finally, conclusions are given in Sect. 5.

\section{Preliminaries and model description}

In this section, some definitions and lemmas are recalled, which will be needed later.

Definition $1([35,36])$ The Riemann-Liouville fractional integral of the function $w(t)$ is defined by

$$
{ }_{t_{0}} I_{t}^{q} w(t)=\frac{1}{\Gamma(q)} \int_{t_{0}}^{t} \frac{w(\zeta)}{(t-\zeta)^{1-q}} d \zeta, \quad q>0
$$


Definition $2([35,36])$ The Caputo fractional derivative of function $w(t)$ is defined by

$$
{ }_{t_{0}}^{c} D_{t}^{q} w(t)=\frac{1}{\Gamma(1-q)} \int_{t_{0}}^{t} \frac{w^{\prime}(\zeta)}{(t-\zeta)^{q}} d \zeta, \quad 0<q<1
$$

Next, we consider a FOCVNN described by

$$
{ }_{t_{0}}^{c} D_{t}^{q} w_{j}(t)=-c_{j} w_{j}(t)+\sum_{p=1}^{n} a_{j p} f_{p}\left(w_{p}(t)\right)+I_{j}(t), \quad j=\Lambda,
$$

or in a compact form

$$
{ }_{t_{0}}^{c} D_{t}^{q} w(t)=-C w(t)+A f(w(t))+I(t)
$$

where $0<q<1, w(t)=\left(w_{1}(t), w_{2}(t), \ldots, w_{n}(t)\right)^{T} \in \mathbb{C}^{n}$ is the state vector, $C=\operatorname{diag}\left(c_{1}, c_{2}\right.$, $\left.\ldots, c_{n}\right) \in \mathbb{R}^{n \times n}$ is the self-feedback connection weight matrix with $c_{j}>0, j \in \Lambda, A=$ $\left(a_{j p}\right)_{n \times n} \in \mathbb{C}^{n \times n}$ is the connection weight matrix, $I(t)=\left(I_{1}(t), I_{2}(t), \ldots, I_{n}(t)\right)^{T} \in \mathbb{C}^{n}$ is the external input vector, $f(w(t))=\left(f_{1}\left(w_{1}(t)\right), f_{2}\left(w_{2}(t)\right), \ldots, f_{n}\left(w_{n}(t)\right)\right)^{T}: \mathbb{C}^{n} \rightarrow \mathbb{C}^{n}$ denotes the vector-valued activation function, and the activation $f_{p}(\cdot)$ satisfies the following assumption.

Assumption 1 For any $w, u \in \mathbb{C}$, there exists a positive constant $l_{p}$ satisfying

$$
\left|f_{p}(z)-f_{p}(w)\right| \leq l_{p}|z-w|
$$

For simplicity, we refer to FOCVNN (2) as the drive system, and the controlled response system is given as

$$
{ }_{t_{0}}^{c} D_{t}^{q} z_{j}(t)=-c_{j} z_{j}(t)+\sum_{p=1}^{n} a_{j p} f_{p}\left(z_{p}(t)\right)+I_{j}(t)+u_{j}(t), \quad 1 \leq j \leq l,
$$

or, in a compact form,

$$
{ }_{t_{0}}^{c} D_{t}^{q} z(t)=-C z(t)+A f(z(t))+I(t)+u(t)
$$

where $z(t)=\left(z_{1}(t), z_{2}(t), \ldots, z_{n}(t)\right)^{T} \in \mathbb{C}^{n}$ is the state vector, $u(t)=\left(u_{1}(t), u_{2}(t), \ldots, u_{n}(t)\right)^{T} \in$ $\mathbb{C}^{n}$ is the control input vector.

Now, we introduce some lemmas, which will be used in the proof of our main results.

Lemma $1([35,36])$ If $w(t) \in \mathbb{C}^{n}\left(\left[t_{0},+\infty\right), \mathbb{R}\right)$, then

$$
{ }_{t_{0}} I_{t t_{0}}^{q_{c}} D_{t}^{q} w(t)=w(t)-\sum_{m=0}^{n-1} \frac{w^{(m)}\left(t_{0}\right)}{m !}\left(t-t_{0}\right)^{m} .
$$

In particular, if $0<q<1$,

$$
t_{0} I_{t t_{0}}^{q c} D_{t}^{q} w(t)=w(t)-w\left(t_{0}\right) .
$$


Lemma 2 ([37]) Let $z(t) \in \mathbb{C}^{n}$ be a differentiable complex-valued vector, then the following inequality holds:

$$
{ }_{t_{0}}^{c} D_{t}^{q}\left(z^{H}(t) P z(t)\right) \leq z^{H}(t) P_{t_{0}}^{c} D_{t}^{q} z(t)+\left({ }_{t_{0}}^{c} D_{t}^{q} z^{H}(t)\right) P z(t),
$$

where $q \in(0,1], t \geq t_{0}, P \in \mathbb{C}^{n \times n}>0$.

Lemma 3 ([38]) Suppose function $\sigma(t)$ is nondecreasing and differentiable on $t \in\left[t_{0}, \infty\right)$, then, for any constant $\mu$ and $t \in\left[t_{0}, \infty\right]$,

$$
{ }_{t_{0}}^{c} D_{t}^{q}(\sigma(t)-\mu)^{2} \leq 2(\sigma(t)-\mu)_{t_{0}}^{c} D_{t}^{q} \sigma(t),
$$

where $0<q<1$.

Lemma 4 ([39]) Assume that $B$ and $G$ are $n \times n$ Hermitian matrices. Let $p_{1} \geq p_{2} \geq \cdots \geq$ $p_{n}, h_{1} \geq h_{2} \geq \cdots \geq h_{n}, r_{1} \geq r_{2} \geq \cdots \geq r_{n}$ be eigenvalues of $B, G$, and $B+G$, respectively. Then one has $p_{i}+h_{n} \leq r_{i} \leq p_{i}+h_{1}, i=1,2, \ldots, n$.

Lemma 5 ([39]) For a symmetric matrix $W \in \mathbb{R}^{n \times n}$ and a diagonal matrix $K=\operatorname{diag}\left(k_{1}, k_{2}\right.$, $\ldots, k_{l}, \underbrace{0,0, \ldots, 0}_{n-l})$ with $k_{i}>0, i=1,2, \ldots, l(1 \leq l<n)$, let $W-K=\left(\begin{array}{cc}B-\tilde{K} & G \\ G^{T} & W_{l}\end{array}\right)$, where $W_{l}$ is the minor matrix of $W$ by removing its first $l$ row-column pairs, $B$ and $G$ are matrices with appropriate dimensions, $\tilde{K}=\operatorname{diag}\left(k_{1}, k_{2}, \ldots, k_{l}\right)$. If $k_{i}>\lambda_{\max }\left(B-G W_{l}^{-1} G^{T}\right), i=1,2, \ldots, l$, $W-K<0$ is equivalent to $W_{l}<0$.

Lemma $6([40])$ Let $V(t)$ be a continuous function on $\left[t_{0},+\infty\right)$ satisfying

$$
{ }_{t_{0}}^{c} D_{t}^{q} V(t) \leq \zeta V(t)
$$

where $0<q<1, \zeta \in \mathbb{R}$ and $t_{0}$ is the initial time. Then

$$
V(t) \leq V\left(t_{0}\right) E_{q}\left[\zeta\left(t-t_{0}\right)^{q}\right]
$$

\section{Main results}

In this section, the partial adaptive control and partial linear control strategies are employed to reduce the control costs, and some novel criteria are derived to ensure the global synchronization of FOCVNNs (2) and (4).

Define the error $e_{j}(t)=u_{j}(t)-w_{j}(t)$ for $j \in \Lambda$, and design the partial adaptive controller $u_{j}(t)$ as follows:

$$
\begin{cases}u_{j}(t)=-\xi_{j}(t) e_{j}(t), & 1 \leq j \leq l, 1 \leq l \leq n-1, \\ c & D_{t}^{q} \xi_{j}(t)=\eta_{j} \overline{e_{j}(t)} e_{j}(t), \\ t_{0}(t)=0, & l+1 \leq j \leq n,\end{cases}
$$

where $\eta_{j}>0, \xi_{j}(t) \in \mathbb{R}$.

Remark 1 Let $\xi_{j}\left(t_{0}\right) \geq 0, j \in \Lambda$, obviously, it follows from the second equality of (5) that $\xi_{j}(t)=\xi_{j}\left(t_{0}\right)+t_{0} I_{t}^{q}\left(\eta_{j} \overline{e_{j}(t)} e_{j}(t)\right) \geq \xi_{j}\left(t_{0}\right)$, thus we can easily derive $\xi_{j}(t) \geq 0$. 
Remark 2 When the FOCVNN (1) and the controlled FOCVNN (3) achieve the global synchronization, the adaptive control gain $\xi_{j}(t)$ tends to some positive constant, this is because the Caputo derivative of a constant is equal to zero

According to (2) and (4) as well as (5), we derive the error system

$$
\begin{aligned}
& { }_{t_{0}}^{c} D_{t}^{q} e(t)=-C e(t)+A[f(z(t))-f(w(t))]-D_{\xi}(t) e(t), \\
& D_{\xi}(t)=\operatorname{diag}(\xi_{1}(t), \xi_{2}(t), \ldots, \xi_{l}(t), \underbrace{0,0, \ldots, 0}_{n-l}) .
\end{aligned}
$$

Theorem 1 Under Assumption 1 and the partial adaptive controller (5), FOCVNNs (2) and (4) can achieve the global synchronization if

$$
\max _{l+1 \leq j \leq n}\left\{l_{j}^{2}-2 c_{j}\right\}+\lambda_{\max }\left(A A^{H}\right)<0
$$

where $\left(A A^{H}\right)_{l}$ is the minor matrix of matrix $A A^{H}$ by removing its first $l(1 \leq l<n)$ rowcolumn pairs.

Proof Construct a Lyapunov function in the following form:

$$
V_{1}(t)=e^{H}(t) e(t)+\sum_{j=1}^{l} \frac{1}{\eta_{j}}\left(\xi_{j}(t)-\xi_{j}^{\star}\right)^{2},
$$

where $\xi_{j}^{\star}$ is a positive constant to be determined.

Calculating the derivative of (8) along the trajectories of (6), we obtain

$$
\begin{aligned}
{ }_{t_{0}}^{c} D_{t}^{q} V_{1}(t) \leq & e^{H}(t)_{t_{0}}^{c} D_{t}^{q} e(t)+\left({ }_{t_{0}}^{c} D_{t}^{q} e^{H}(t)\right) e(t)+\sum_{j=1}^{l} \frac{2}{\eta_{j}}\left(\xi_{j}(t)-\xi_{j}^{\star}\right)_{t_{0}}^{c} D_{t}^{q} \xi_{j}(t) \\
= & e^{H}(t)\left(-C e(t)+A[f(z(t))-f(w(t))]-D_{\xi}(t) e(t)\right) \\
& +\left(-C e(t)+A[f(z(t))-f(w(t))]-D_{\xi}(t) e(t)\right)^{H} e(t) \\
& +2 \sum_{j-1}^{l}\left(\xi_{j}(t)-\xi_{j}^{\star}\right) \\
= & -e^{H}(t)\left(C+C^{H}\right) e(t)+e^{H}(t) A(f(z(t))-f(u(t))) \\
& +(f(z(t))-f(u(t)))^{H} A^{H} e(t)-2 e^{H}(t) D_{\xi}^{\star} e(t) \\
\leq & -2 e^{H}(t) C e(t)+\lambda_{\max }\left(A A^{H}\right) e^{H}(t) e(t) \\
& +(f(z(t))-f(u(t)))^{H}(f(z(t))-f(u(t)))-2 e^{H}(t) D_{\xi}^{\star} e(t)
\end{aligned}
$$

where $D_{\xi}^{\star}=\operatorname{diag}(\xi_{1}^{\star}, \xi_{2}^{\star}, \ldots, \xi_{l}^{\star}, \underbrace{0,0, \ldots, 0}_{n-l})$. From Assumption 1, we can obtain

$$
(f(z(t))-f(u(t)))^{H}(f(z(t))-f(u(t))) \leq e^{H}(t) L L e(t),
$$


where $L=\operatorname{diag}\left(l_{1}, l_{2}, \ldots, l_{n}\right)$ is the real-valued positive diagonal matrix. According to (9) and (10), we have

$$
{ }_{t_{0}}^{c} D_{t}^{q} V_{1}(t) \leq e^{H}(t)\left(W-2 D_{\xi}^{\star}\right) e(t)
$$

where $W=\lambda_{\max }\left(A A^{H}\right) I_{n \times n}+L L-2 C$. Using matrix decomposition, we have

$$
W-2 D_{\xi}^{\star}=\left(\begin{array}{cc}
B-2 \tilde{D} & G \\
G^{H} & W_{l}
\end{array}\right),
$$

where $B=\left(b_{i j}\right)_{l \times l}, b_{i j}=w_{i j}, i, j=1,2, \ldots, l, \tilde{D}=\operatorname{diag}\left(\xi_{1}^{\star}, \xi_{2}^{\star}, \ldots, \xi_{l}^{\star}\right), G=\left(g_{i j}\right)_{l \times(n-l)}, g_{i j}=w_{i j}$, $i=1,2, \ldots, l, j=l+1, l+2, \ldots, n$, and $W_{l}$ is the minor matrix of $W$ by removing its first $l(1 \leq l \leq n-1)$ row-column pairs. It follows from Lemma 4 and condition (7) that $\lambda_{\max }\left(\left(\lambda_{\max }\left(A A^{H}\right) I_{n \times n}+L L-2 C\right)_{l}\right) \leq \lambda_{\max }\left((L L-2 C)_{l}\right)+\lambda_{\max }\left(A A^{H}\right)<0$, which implies that $W_{l}<0$. If we choose positive constants $\xi_{j}^{\star}>0, i=1,2, \ldots, l$, such that $\xi_{j}^{\star}>$ $\frac{1}{2} \lambda_{\max }\left(B-G W_{l}^{-1} G^{H}\right)$, according to Lemma 5 and $W_{l}<0$, we derive $W-2 D_{\xi}^{\star}<0$, then it follows from (11) that

$$
{ }_{t_{0}}^{c} D_{t}^{q} V_{1}(t) \leq-\lambda^{\star} e^{H}(t) e(t)
$$

where $-\lambda^{\star}=\lambda_{\max }\left(W-2 D_{\xi}^{\star}\right)$ and $\lambda^{\star}>0$. According to (12), there exists a nonnegative function $r(t)$ such hat

$$
{ }_{t_{0}}^{c} D_{t}^{q} V_{1}(t)+r(t)=-\lambda^{\star} e^{H}(t) e(t) .
$$

Integrating both sides of (13) from $t_{0}$ to $t$, we obtain

$$
\begin{aligned}
-\lambda^{\star} \int_{t_{0}}^{t} e^{H}(\zeta) e(\zeta) d \zeta= & \int_{t_{0}}^{t}{ }_{t_{0}} D_{\zeta}^{q} V_{1}(\tau) d \zeta+\int_{t_{0}}^{t} r(\zeta) d \zeta \\
& =\frac{1}{\Gamma(1-q)} \int_{t_{0}}^{t} \int_{t_{0}}^{\zeta} \frac{V_{1}^{\prime}(\tau)}{(\zeta-\tau)^{q}} d \tau d \zeta+\int_{t_{0}}^{t} r(\zeta) d \zeta \\
& =\frac{1}{\Gamma(1-q)} \int_{t_{0}}^{t} \int_{\tau}^{t} \frac{V_{1}^{\prime}(\tau)}{(\zeta-\tau)^{q}} d \zeta d \tau+\int_{t_{0}}^{t} r(\zeta) d \zeta \\
& =\frac{1}{(1-q) \Gamma(1-q)} \int_{t_{0}}^{t} V_{1}^{\prime}(\tau)(t-\tau)^{1-q} d \tau+\int_{t_{0}}^{t} r(\zeta) d \zeta \\
& =-\frac{V_{1}\left(t_{0}\right)\left(t-t_{0}\right)^{1-q}}{\Gamma(2-q)}+\frac{1}{\Gamma(1-q)} \int_{t_{0}}^{t} V_{1}(\tau)(t-\tau)^{-q} d \tau \\
& +\int_{t_{0}}^{t} r(\zeta) d \zeta \\
\geq & -\frac{V_{1}\left(t_{0}\right)\left(t-t_{0}\right)^{1-q}}{\Gamma(2-q)}
\end{aligned}
$$

it is easy to obtain from (14) that

$$
\int_{t_{0}}^{t} e^{H}(\zeta) e(\zeta) d \zeta \leq \frac{V_{1}\left(t_{0}\right)\left(t-t_{0}\right)^{1-q}}{\lambda^{\star} \Gamma(2-q)}
$$


hence

$$
\lim _{t \rightarrow+\infty} \frac{\int_{t_{0}}^{t} e^{H}(\zeta) e(\zeta) d \zeta}{\left(t-t_{0}\right)^{1-q}} \leq \frac{V\left(t_{0}\right)}{\lambda^{\star} \Gamma(2-q)}
$$

By employing the L'Hospital rule, we get

$$
\lim _{t \rightarrow+\infty} e^{H}(t) e(t)\left(t-t_{0}\right)^{q} \leq \frac{V_{1}\left(t_{0}\right)}{\lambda^{\star} \Gamma(2-q)} .
$$

Taking fractional integration of (13) from $t_{0}$ to $t$ one derives

$$
\begin{aligned}
V_{1}(t)-V_{1}\left(t_{0}\right) & =-\frac{1}{\Gamma(q)} \int_{t_{0}}^{t} \frac{r(\zeta)}{(t-\zeta)^{1-q}} d \zeta-\frac{\lambda^{\star}}{\Gamma(q)} \int_{t_{0}}^{t} \frac{e^{H}(\zeta) e(\zeta)}{(t-\zeta)^{1-q}} d \zeta \\
& =0
\end{aligned}
$$

Combining (8) and (18) yields

$$
e^{H}(t) e(t) \leq V_{1}(t) \leq V_{1}\left(t_{0}\right)
$$

that is, $e^{H}(t) e(t)$ must be bounded. Together with (17) and (19), we know that there exists a $t_{1}>0$ satisfying

$$
e^{H}(t) e(t) \leq \frac{V_{1}\left(t_{0}\right)}{\lambda^{\star} \Gamma(2-q)\left(t-t_{0}\right)^{q}}
$$

for all $t \geq t_{1}$, which implies

$$
\lim _{t \rightarrow+\infty} e^{H}(t) e(t)=0
$$

this indicates that the controlled response FOCVNN (4) is synchronized with FOCVNN (2) under the partial adaptive controller (5).

If we take $\eta_{j}=0$ in controller (5), then the partial adaptive controller (5) degenerates into the partial linear feedback controller

$$
\begin{cases}u_{j}(t)=-\xi_{j} e_{j}(t), & 1 \leq j \leq l, 1 \leq l \leq n-1, \\ u_{j}(t)=0, & l+1 \leq j \leq n\end{cases}
$$

where $\xi_{j} \in \mathbb{R}$. In this case, we can derive the following corollary.

Corollary 1 Under Assumption 1 and condition (7), if the algebraic inequality

$$
\min _{1 \leq j \leq l}\left\{\xi_{j}\right\}>\frac{1}{2} \lambda_{\max }\left(B-G W_{l}^{-1} G^{H}\right)
$$

is satisfied, then FOCVNNs (2) and (4) can achieve the global Mittag-Leffler synchronization under the partial linear feedback controller (20). 
Proof Construct a Lyapunov function in the following form:

$$
V_{2}(t)=e^{H}(t) e(t)
$$

Using a similar process with respect to (9)-(12), we derive

$$
{ }_{t_{0}}^{c} D_{t}^{q} V_{2}(t) \leq-\hat{\lambda}_{1} V_{2}(t)
$$

where $-\hat{\lambda}_{1}=\lambda_{\max }\left(W-2 D_{\xi}\right), W=\lambda_{\max }\left(A A^{H}\right) I_{n \times n}+L L-2 C, D_{\xi}=\operatorname{diag}\left(\xi_{1}, \xi_{2}, \ldots, \xi_{l}\right.$, $\underbrace{0,0, \ldots, 0}_{n-l})$. According to Lemma 6 , we have $V(t) \leq V\left(t_{0}\right) E_{q}\left(-\hat{\lambda}_{1}\left(t-t_{0}\right)^{q}\right)$, the proof of Corollary 1 is completed.

Remark 3 We observe that the existing control schemes for neural networks almost control all neurons, we realize synchronization for the addressed FOCVNNs in this paper by employing partial linear control and partial adaptive control schemes.

If we take $l=n$, that is, all neurons are controlled, then the partial adaptive controller (5) becomes the adaptive controller

$$
\left\{\begin{array}{l}
u_{j}(t)=-\xi_{j}(t) e_{j}(t), \\
c_{0} D_{t}^{q} \xi_{j}(t)=\eta_{j} \overline{e_{j}(t)} e_{j}(t),
\end{array}\right.
$$

where $\eta_{j}>0, \xi_{j}(t) \in \mathbb{R}$. In this case, we can derive the following corollary.

Corollary 2 Under Assumption 1, FOCVNNs (2) and (4) can achieve the global synchronization under the adaptive controller (23).

Proof Construct a Lyapunov function in the following form:

$$
V_{3}(t)=e^{H}(t) e(t)+\sum_{j=1}^{n} \frac{1}{\eta_{j}}\left(\xi_{j}(t)-\xi_{j}^{\star}\right)^{2}
$$

where $\xi_{j}^{\star}$ is a positive constant to be determined.

Calculating the derivative of (24) along the trajectories of (6), we obtain

$$
\begin{aligned}
{ }_{t_{0}}^{c} D_{t}^{q} V(t) \leq & e^{H}(t)_{t_{0}}^{c} D_{t}^{q} e(t)+\left({ }_{t_{0}}^{c} D_{t}^{q} e^{H}(t)\right) e(t)+\sum_{j=1}^{n} \frac{2}{\eta_{j}}\left(\xi_{j}(t)-\xi_{j}^{\star}\right)_{t_{0}}^{c} D_{t}^{q} \xi_{j}(t) \\
= & -e^{H}(t)\left(C+C^{H}\right) e(t)+e^{H}(t) A(f(z(t))-f(u(t))) \\
& +(f(z(t))-f(u(t)))^{H} A^{H} e(t)-2 e^{H}(t) \widetilde{D}_{\xi}^{\star} e(t) \\
\leq & e^{H}(t)\left(A A^{H}+L L-2 C-2 \widetilde{D}_{\xi}^{\star}\right) e(t) \\
\leq & \hat{\lambda}_{2} e^{H}(t) e(t),
\end{aligned}
$$

where $\widetilde{D}_{\xi}^{\star}=\operatorname{diag}\left(\xi_{1}^{\star}, \xi_{2}^{\star}, \ldots, \xi_{n}^{\star}\right)$. We choose $\xi_{j}^{\star}>\frac{1}{2} \lambda_{\max }\left(A A^{H}+L L-2 C\right), j \in \Lambda$, which implies that $A A^{H}+L L-2 C-2 \widetilde{D}_{\xi}^{\star}<0$, let $-\hat{\lambda}_{2}=\lambda_{\max }\left(A A^{H}+L L-2 C-2 \widetilde{D}_{\xi}^{\star}\right)$. Using a similar 
proof with respect to (13)-(19), we derive $\lim _{t \rightarrow+\infty} e^{H}(t) e(t)=0$, the proof of Corollary 2 is completed.

Remark 4 Evidently, Theorem 1, Corollaries 1 and 2 still hold for $q=1$.

Remark 5 In [23, 29-31], the authors obtained synchronization criteria by real decomposition method. Compared with the real decomposition method in [23, 29-31], the Lyapunov direct method employed in this paper is more nature and compact. In [23], the authors adopted adaptive feedback control strategy, and realized synchronization for the considered FOCVNNs. In [32], the authors employed linear feedback control strategy, and achieved synchronization for the considered FOCVNNs. Compared with control strategies in $[23,32]$, our partial adaptive control strategy is more easy and has less cost from the point of view of practical applications.

\section{Numerical simulations}

In this section, some numerical simulations are presented to illustrate the effectiveness of our results obtained in the previous section.

Consider a FOCVNN consisting of four neurons described by

$$
{ }_{0}^{c} D_{t}^{q} w_{j}(t)=-c_{j} w_{j}(t)+\sum_{p=1}^{4} a_{j p} f_{p}\left(w_{p}(t)\right)+I_{j}(t), \quad j=1,2,3,4,
$$

where $q=0.98, w_{j}(t)=w_{j}^{R}(t)+i w_{j}^{I}(t)$ with $w_{j}^{R}(t), w_{j}^{I}(t) \in \mathbb{R}$, the initial state $w_{1}(0)=0.5+$ $0.1 i, w_{2}(0)=-0.6-0.3 i, w_{3}(0)=0.4+0.2 i$, and $w_{4}(0)=0.1+0.2 i$. In addition, $f_{p}\left(w_{p}(t)\right)=$ $\tanh \left(w_{p}^{R}(t)\right)+i \tanh \left(w_{p}^{I}(t)\right), I_{1}(t)=I_{2}(t)=I_{3}(t)=I_{4}(t)=0, C=\operatorname{diag}(1,1,12.5,12.5)$, and

$$
A=\left(\begin{array}{cccc}
2-2 i & -1.2+1.2 i & 0 & -0.6-0.4 \\
1.8+1.8 i & 1.71+1.71 i & 1.15+1.15 i & 0.4+0.1 i \\
-1.75-1.75 i & 0 & 0.1+0.1 i & 0.5+i \\
1+0.2 i & -1+0.1 i & 0.1+0.2 i & 0.2+0.1 i
\end{array}\right)
$$

By simple calculation, we derive that Assumption 1 is satisfied with $l_{p}=1, j=1,2,3,4$. Figures 1 and 2 depict the phase trajectories of real and imaginary parts with respect to the state variables of FOCVNN (26), respectively.
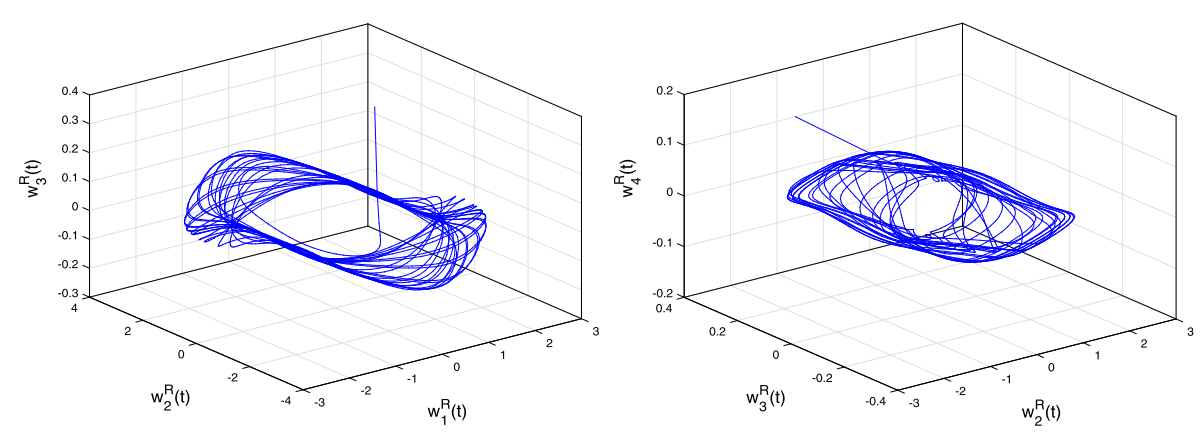

Figure 1 Real part of state variables of FOCVNN (26) 

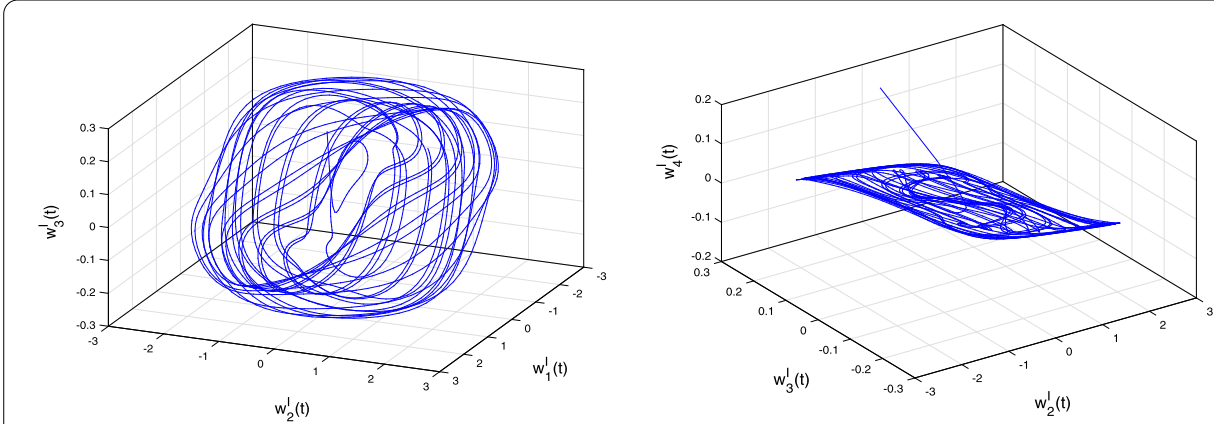

Figure 2 Imaginary part of state variables of FOCVNN (26)

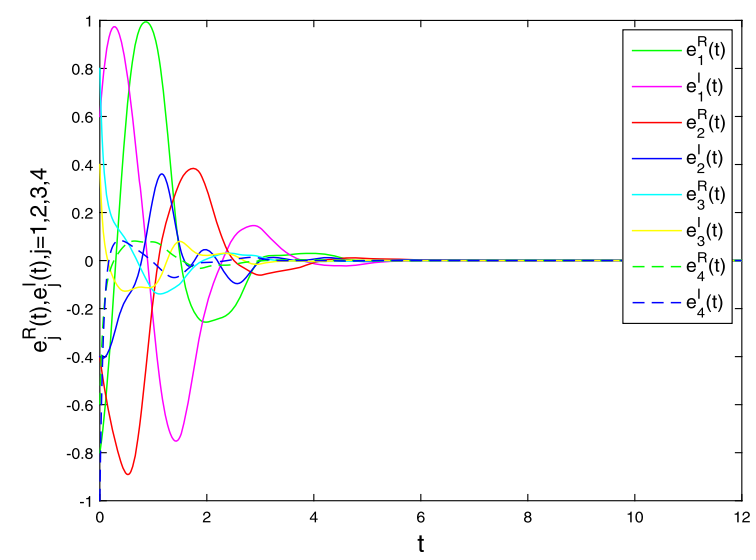

Figure 3 Time response curves of errors $e_{j}^{R}(t)$ and $e_{j}^{\prime}(t)$ under the partial adaptive controller (5)

The response system is given by

$$
{ }_{0}^{c} D_{t}^{q} z_{j}(t)=-c_{j} z_{j}(t)+\sum_{p=1}^{4} a_{j p} f_{p}\left(z_{p}(t)\right)+I_{j}(t)+u_{j}(t), \quad j=1,2,3,4,
$$

where the initial state is selected as $z_{1}(0)=-0.3+0.7 i, z_{2}(0)=-1-0.7 i, z_{3}(0)=1.2+0.6 i$, $z_{4}(0)=-0.5-0.8 i$, and the other parameters of FOCVNN (27) are the same as that of FOCVNN (26). In the simulation, we only control the first two neurons of FOCVNN (27).

If we take $q=0.98, \eta_{1}=\eta_{2}=0.36, \xi_{1}(0)=0.4, \xi_{2}(0)=0.6$ in the partial adaptive controller (5). By simple calculation, we get

$$
\max _{3 \leq j \leq 4}\left\{l_{j}^{2}-2 \operatorname{Re}\left(c_{j}\right)\right\}+\lambda_{\max }\left(A A^{H}\right)=-0.8270<0
$$

then condition (7) is satisfied. On the basis of Theorem 1, the FOCVNN (26) and the controlled FOCVNN (27) can achieve the global synchronization under the partial adaptive controller (5), which is depicted in Fig. 3. As shown in Fig. 3, the state trajectories of errors $e_{j}^{R}(t)$ and $e_{j}^{I}(t)$ converge to zero. The time response trajectories of $\xi_{1}(t)$ and $\xi_{2}(t)$ are depicted in Fig. 4, we can see from Fig. 4 that the adaptive control gains $\xi_{1}(t)$ and $\xi_{2}(t)$ converge to some positive constants, which is in accordance with Remark 2. 


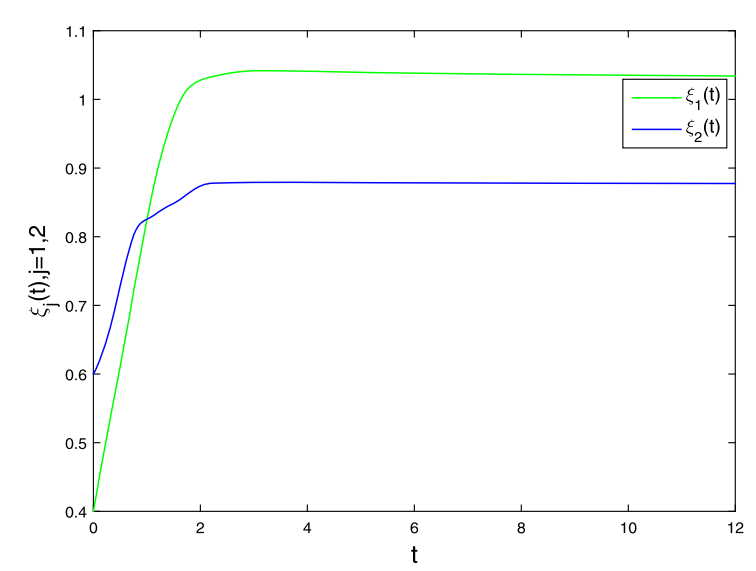

Figure 4 Time response curves of $\xi_{1}(t)$ and $\xi_{2}(t)$

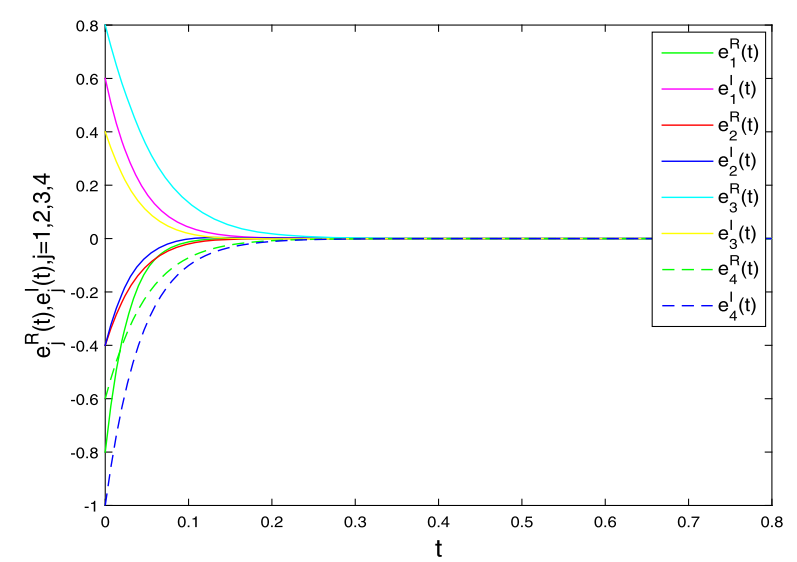

Figure 5 Time response curves of errors $e_{j}^{R}(t)$ and $e_{j}^{\prime}(t)$ under the partial linear controller (20)

If we set $\xi_{1}=\xi_{2}=11.3$ in the partial linear controller (20), by simple calculation,

$$
11.3=\min _{1 \leq j \leq l}\left\{\xi_{j}\right\}>\frac{1}{2} \lambda_{\max }\left(B-G W_{l}^{-1} G^{H}\right)=11.0865 .
$$

According to Corollary 1, the FOCVNN (26) and the controlled FOCVNN (27) can achieve the global synchronization under the partial linear controller (20), which is depicted in Fig. 5, we can observe from Fig. 5 that the state trajectories of errors $e_{j}^{R}(t)$ and $e_{j}^{I}(t)$ converge to zero.

If we set $q=0.98, \eta_{1}=\eta_{2}=\eta_{3}=\eta_{4}=0.36, \xi_{1}(0)=0.4, \xi_{2}(0)=0.6, \xi_{1}(0)=0.5, \xi_{2}(0)=0.3$ in the adaptive controller (23). The evolutions of synchronization errors $e_{j}^{R}(t)$ and $e_{j}^{I}(t)$ are shown in Fig. 6, we can observe from Fig. 6 that the controlled FOCVNN (27) can synchronize with the FOCVNN (26) under the adaptive controller (23). Figure 7 shows the time response trajectories of $\xi_{1}(t), \xi_{2}(t), \xi_{3}(t)$ and $\xi_{4}(t)$. 


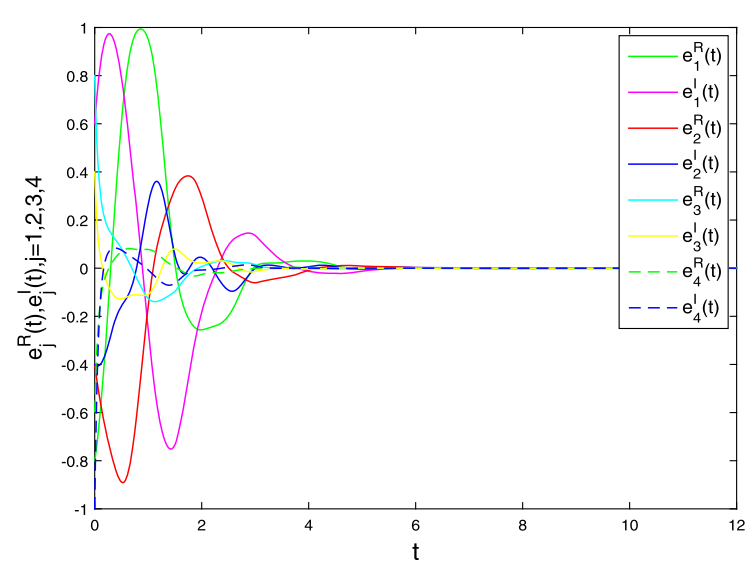

Figure 6 Time response curves of errors $e_{j}^{R}(t)$ and $e_{j}^{\prime}(t)$ under the adaptive controller (23)

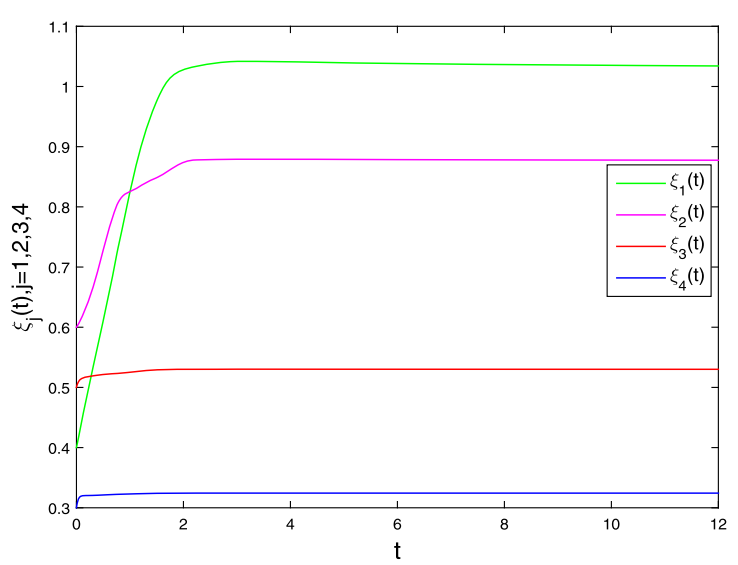

Figure 7 Time response curves of $\xi_{j}(t), j=1,2,3,4$

\section{Conclusion}

This paper is concerned with the global synchronization of FOCVNNs. To realize the synchronization goal, the partial adaptive controller and partial linear feedback controller are designed, respectively. On the basis of the Lyapunov method, the L'Hospital rule and some complex analysis techniques, some succinct criteria are derived to ensure the global synchronization for the considered FOCVNNs. Numerical simulations are given to show the effectiveness and feasibility of the proposed method. The topic concerning dynamics analysis for FOCVNNs is of importance, we will make some efforts on the finite-time synchronization of FOCVNNs.

\section{Acknowledgements}

The authors are grateful to the editors and anonymous referees for their valuable suggestions and comments, which greatly improved the presentation of this paper.

\section{Funding}

This work is supported by the National Natural Science Foundation of China (Grant Nos. 11702237, 11861065), Project Funded by China Postdoctoral Science Foundation (Grant No. 1107010238), the Natural Science Foundation of Xinjiang (Grant No. 2017D01C082), the Scientific Research Program of the Higher Education Institution of Xinjiang (Grant No. XJEDU2017S001), and the Doctoral Scientific Research Foundation of Xinjiang University (Grant No. BS160204). 
Availability of data and materials

Not applicable.

\section{Competing interests}

The authors declare that they have no competing interests.

\section{Authors' contributions}

All authors contributed equally to this work. All authors read and approved the final manuscript.

\section{Publisher's Note}

Springer Nature remains neutral with regard to jurisdictional claims in published maps and institutional affiliations.

Received: 18 March 2020 Accepted: 29 June 2020 Published online: 23 July 2020

\section{References}

1. Balasubramaniam, P., Rakkiyappan, R.: Global asymptotic stability of stochastic recurrent neural networks with multiple discrete delays and unbounded distributed delays. Appl. Math. Comput. 204, 680-686 (2008)

2. Tang, R., Yang, X., Wan, X.: Finite-time cluster synchronization for a class of fuzzy cellular neural networks via non-chattering quantized controllers. Neural Netw. 113, 79-90 (2019)

3. Qiu, J., Cao, J.: Delay-dependent exponential stability for a class of neural networks with time delays and reaction-diffusion terms. J. Franklin Inst. 346, 301-314 (2009)

4. Shen, H., Zhu, Y., Zhang, L., Park, J.: Extended dissipative state estimation for Markov jump neural networks with unreliable links. IEEE Trans. Neural Netw. Learn. Syst. 28, 346-358 (2017)

5. Wu, X., Tang, Y., Zhang, W.: Stability analysis of switched stochastic neural networks with time-varying delays. Neural Netw. 51, 39-49 (2014)

6. Xu, W., Cao, J., Xiao, M., Ho, D., Wen, G.: A new framework for analysis on stability and bifurcation in a class of neural networks with discrete and distributed delays. IEEE Trans. Cybern. 45, 2224-2236 (2015)

7. Pecora, L., Carroll, T.: Synchronization in chaotic systems. Phys. Rev. Lett. 64, 821-824 (1990)

8. Gan, Q., Xu, R., Kang, X.: Synchronization of chaotic neural networks with mixed time delays. Commun. Nonlinear Sci. Numer. Simul. 16, 966-974 (2011)

9. Chen, S., Cao, J.: Projective synchronization of neural networks with mixed time-varying delays and parameter mismatch. Nonlinear Dyn. 67, 1397-1406 (2012)

10. Tang, Z., Park, J., Feng, J.: Impulsive effects on quasi-synchronization of neural networks with parameter mismatches and time-varying delay. IEEE Trans. Neural Netw. Learn. Syst. 29, 908-919 (2018)

11. Wu, A., Wen, S., Zeng, Z:: Synchronization control of a class of memristor-based recurrent neural networks. Inf. Sci. $183,106-116(2012)$

12. Sun, Y., Wei, Z., Sun, G., Ju, P., Wei, Y.: Stochastic synchronization of nonlinear energy resource system via partial feedback control. Nonlinear Dyn. 70, 2269-2278 (2012)

13. Zhang, H., Xie, Y., Wang, Z., Zheng, C.: Adaptive synchronization between two different chaotic neural networks with time delay. IEEE Trans. Neural Netw. 18, 1841-1845 (2007)

14. Li, X., Rakkiyappan, R., Velmurugan, G.: Dissipayivity analysis of memristor-based complex-valued neural networks with time-varying delay. Inf. Sci. 294, 645-665 (2015)

15. Ding, X., Cao, J., Alsaedi, A., Alsaadi, F., Hayat, T.: Robust fixed-time synchronization for uncertain complex-valued neural networks with discontinuous activation functions. Neural Netw. 90, 42-55 (2017)

16. Wu, Z., Chen, G., Fu, X.: Synchronization of a network coupled with complex-variable chaotic systems. Chaos 22 Article ID 023127 (2012)

17. Hu, B., Song, Q., Li, K., Zhao, Z., Liu, Y., Alsaadi, F.: Global $\mu$-synchronization of impulsive complex-valued neural networks with leakage delay and mixed time-varying delays. Neurocomputing 307, 106-116 (2018)

18. Song, Q., Yan, H., Zhao, Z., Liu, Y.: Global exponential stability of complex-valued neural networks with both time-varying delays and impulsive effects. Neural Netw. 79, 108-116 (2016)

19. Yildiz, T., Jajarmi, A., Yildiz, B., Baleanu, D.: New aspects of time fractional optimal control problems within operators with nonsingular kernel. Discrete Contin. Dyn. Syst., Ser. S 13, 407-428 (2020)

20. Baleanu, D., Jajarmi, A., Mohammadi, H., Rezapour, S.: A new study on the mathematical modelling of human liver with Caputo-Fabrizio fractional derivative. Chaos Solitons Fractals 134. Article ID 109705 (2020)

21. Li, H., Muhammadhaji, A., Zhang, L., Teng, Z.: Stability analysis of a fractional-order predator prey model incorporating a constant prey refuge and feedback control. Adv. Differ. Equ. 2018, Article ID 325 (2018)

22. Jajarmi, A., Arshad, S., Baleanu, D.: A new fractional modelling and control strategy for the outbreak of dengue fever. Physica A 535, Article ID 122524 (2019)

23. Li, L., Wang, Z., Lu, J., Li, Y.: Adaptive synchronization of fractional-order complex-valued neural networks with discrete and distributed delays. Entropy 20, Article ID 124 (2018)

24. Baleanu, D., Jajarmi, A., Sajjadi, S., Mozyrska, D.: A new fractional model and optimal control of a tumor-immune surveillance with non-singular derivative operator. Chaos 29, Article ID 083127 (2019)

25. Jajarmi, A., Ghanbari, B., Baleanu, D.: A new and efficient numerical method for the fractional modelling and optimal control of diabetes and tuberculosis co-existence. Chaos 29, Article ID 093111 (2019)

26. Stamova, I: Global Mittag-Leffler stability and synchronization of impulsive fractional-order neural networks with time-varying delays. Nonlinear Dyn. 77, 1251-1260 (2014)

27. Huang, C., Cao, J., Xiao, M., Alsaedi, A., Hayat, T.: Bifurcations in a delayed fractional complex-valued neural network. Appl. Math. Comput. 292, 210-227 (2017)

28. Rakkiyappan, R., Cao, J.: Existence and uniform stability analysis of fractional-order complex-valued neural networks with time delays. IEEE Trans. Neural Netw. Learn. Syst. 26, 84-97 (2015) 
29. Bao, H., Park, J., Cao, J.: Synchronization of fractional-order complex-valued neural networks with time delay. Neural Netw. 81, 16-28 (2016)

30. Yang, X., Li, C., Huang, T., Song, Q., Huang, J.: Synchronization of fractional-order memristor-based complex-valued neural networks with uncertain parameters and time delays. Chaos Solitons Fractals 110, 105-123 (2018)

31. Zhang, W., Zhang, H., Cao, J., Alsaadi, F., Chen, D.: Synchronization in uncertain fractional-order memristive complex-valued neural networks with multiple time delays. Neural Netw. 110, 186-198 (2019)

32. Li, H., Hu, C., Cao, J., Jiang, H., Alsaedi, A.: Quasi-projective and complete synchronization of fractional-order complex-valued neural networks with time delays. Neural Netw. 118, 102-109 (2019)

33. Li, Y.: Impulsive synchronization of stochastic neural networks via controlling partial states. Neural Process. Lett. 46, 59-69 (2017)

34. Wu, A., Zeng, Z., Song, X.: Global Mittag-Leffler stability of fractional-order bidirectional associative memory neural networks. Neurocomputing 117, 489-496 (2016)

35. Kilbas, A., Srivastava, H., Trujillo, J.: Theory and Application of Fractional Differential Equations. Elsevier, New York (2006)

36. Podlubny, l.: Fractional Differential Equations. Academic Press, San Diego (1999)

37. Xu, Q., Zhuang, S., Liu, S., Xiao, J.: Decentralized adaptive coupling synchronization of fractional-order complex-variable dynamical networks. Neurocomputing 186, 119-126 (2016)

38. $\mathrm{Yu}, \mathrm{J}, \mathrm{Hu}, \mathrm{C}$., Jiang, H.: Corrogendum to "Projective synchronization for fractional neural networks". Neural Netw. 67, 152-154 (2015)

39. Song, Q., Shu, H., Zhao, Z., Liu, Y., Alsaadi, F.: Lagrange stability analysis for complex-valued neural networks with leakage delay and mixed time-varying delays. Neurocomputing 244, 33-41 (2017)

40. Li, H., Jiang, Y., Wang, Z., Zhang, L., Teng, Z:: Mittag-Leffler stability of coupled system of fractional-order differential equations on network. Appl. Math. Comput. 270, 269-277 (2015)

\section{Submit your manuscript to a SpringerOpen ${ }^{\circ}$ journal and benefit from:}

- Convenient online submission

- Rigorous peer review

- Open access: articles freely available online

- High visibility within the field

Retaining the copyright to your article

Submit your next manuscript at $\boldsymbol{\nabla}$ springeropen.com 